\title{
Physician Global Assessment of Disease Activity
}

National Cancer Institute

\section{Source}

National Cancer Institute. Physician Global Assessment of Disease Activity. NCI

Thesaurus. Code C121346.

A physician or clinician-scored instrument that estimates the level of activity of a disease at a given time taking into account all disease manifestations. Typically scored on a numeric rating scale or visual analogue scale, or may be recorded using descriptors such as remission, or low, moderate, or high disease activity. 